\title{
Erratum: Universal Lindblad equation for open quantum systems [Phys. Rev. B 102, 115109 (2020)]
}

\author{
Frederik Nathan 1 and Mark S. Rudner \\ (Received 10 August 2021; published 2 September 2021)
}

DOI: 10.1103/PhysRevB.104.119901

After publication, we became aware of a few misprinted indices and signs in equations of the main text and appendices. We provide the corrected expressions below. In addition, we discovered that the code that was used to generate Figs. 1 and 2 contained a bug that rescaled the time axis by a factor of $2 \pi$. We therefore provide the amended figures here. None of these corrections affect the main results or conclusions of our paper. In particular, the corrections do not affect the derivation of the universal Lindblad equation (ULE) and its error bounds.

\section{CORRECTED EXPRESSIONS}

(1) The indices in Eq. (34) should be corrected to read

$$
\Lambda=\sum_{\alpha, \beta, l, m, n} f_{\alpha \beta}\left(E_{l}-E_{m}, E_{n}-E_{l}\right) X_{m l}^{(\alpha)} X_{l n}^{(\beta)}|m\rangle\langle n| .
$$

(2) The expression for $f\left(E_{1}, E_{2}\right)$ below Eq. (34) should read

$$
\boldsymbol{f}\left(E_{1}, E_{2}\right) \equiv-2 \pi \gamma \mathcal{P} \int_{-\infty}^{\infty} d \omega \omega^{-1} \boldsymbol{g}\left(\omega-E_{1}\right) \boldsymbol{g}\left(\omega+E_{2}\right) .
$$

(3) The expression for $c_{n}$ in Eq. (36) should read

$$
c_{n} \equiv \frac{(-i)^{n}}{n !} \int_{-\infty}^{\infty} d t g(t) t^{n}
$$

(4) The expression for $\tilde{L}_{\lambda}^{\dagger}(t)$ below Eq. (C26) should read

$$
\tilde{L}_{\lambda}^{\dagger}(t)=\sqrt{\gamma} \int_{-\infty}^{\infty} d s g_{\alpha \lambda}(s-t) \tilde{X}_{\alpha}(s) .
$$

(5) The definition of $\phi_{\alpha \beta}$ below Eq. (D1) should read: "where $\left\{\phi_{\alpha \beta}(t, s)\right\}$ denote the elements of the matrix $\phi(t, s) \equiv$ $\boldsymbol{g}(t) \boldsymbol{g}(-s) \operatorname{sgn}(t-s) . "$

(6) The definition of $\boldsymbol{f}$ in Eq. (D4) should read

$$
\boldsymbol{f}(p, q)=\frac{\gamma}{2 i} \int_{-\infty}^{\infty} d t \int_{-\infty}^{\infty} d s \operatorname{sgn}(t-s) e^{-i(p t+q s)} \boldsymbol{g}(t) \boldsymbol{g}(-s)
$$

(7) In Eq. (D8) the subscripts should be corrected to

$$
\Lambda=\sum_{m, n, l} \sum_{\alpha \beta} f_{\alpha \beta}\left(E_{l m}, E_{n l}\right) X_{m l}^{(\alpha)} X_{l n}^{(\beta)}|m\rangle\langle n| .
$$

\section{CORRECTED NUMERICAL RESULTS}

As mentioned above, the code that was used to generate Figs. 1 and 2 had a bug that scaled the time arguments of the bath and jump correlators $J(t)$ and $g(t)$ by a factor of $2 \pi$. The corrected version of Fig. 1 of the paper is provided in Fig. E1 here. Due to this bug, the quoted numerical values for $\Gamma$ and $\tau$ were factors of $4 \pi^{2}$ and $2 \pi$ too small throughout the text, respectively. For reference, we provide the explicit corrections below. 


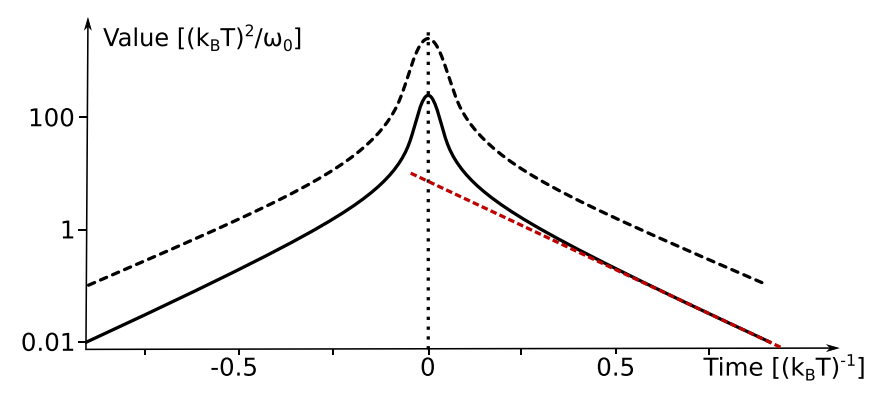

FIG. E1. Absolute value of the jump correlator $g(t)$ (solid) and bath correlation function $J(t)$ (black dashed line) for the Ohmic bath studied in Sec. II B of the original paper with corrected time axis. The red line indicates the fit used to obtain the exponential decay constant for $g$. This figure replaces Fig. 1 in the original paper.

(1) The value of $\tau$ quoted in the final paragraph of Sec. II B should read

$$
\tau=0.043 T^{-1} .
$$

Additionally, the slope of $\ln |g(t)|$ we obtain by linear regression is given by $0.14 T^{-1}$.

(2) In Sec. V A, the values of $\Gamma$ and $\tau$ quoted in the text should be replaced with the following:

$$
\Gamma=16 \eta, \quad \tau=0.00082 \eta^{-1} .
$$

Likewise the values of the products $\Gamma \tau$ and $2 \Gamma^{2} \tau$ quoted in the text should be replaced with $\Gamma \tau=0.013$ and $2 \Gamma^{2} \tau=0.43 \eta$.

(3) In Sec. V B, the values of $\Gamma$ and $\tau$ quoted in the text should be replaced with the following:

$$
\Gamma=141 \eta, \quad \tau=0.020 \eta^{-1} .
$$

The values of the products $\Gamma \tau$ and $2 \Gamma^{2} \tau$ quoted in the text should be replaced with $\Gamma \tau=2.8$ and $2 \Gamma^{2} \tau=803 \eta$.

The purpose of the simulations in Sec. V is to demonstrate the application of the ULE to a spin-chain system [see Fig. E2(a)] in a regime where the error bounds for the ULE indicate that it accurately describes the system $(\Gamma \tau \ll 1$, and $\Gamma^{2} \tau$ is much smaller than the other characteristic energy scales of the system). With the corrected values of $\Gamma$ and $\tau$ above, the parameter sets used in the demonstrations in Sec. $\mathrm{V}$ fall outside the regime where the errors of the ULE are rigorously guaranteed to be small. For reference, we therefore provide complementary data from simulations of the spin chain in Sec. V for parameter sets that clearly satisfy the conditions for the validity of the ULE as a good approximation to the true dynamics and where we observe the same qualitative behavior as in Fig. 2 of the original paper.

Throughout the complementary simulations we set $B_{z}=25 \eta, \Lambda=30 \eta, \omega_{0}=25 \eta$ (for both baths), and $T_{2}=100 \eta$ and $T_{1}=10 \eta$. In Figs. E2(b) and E2(c) we plot the values of $\langle M\rangle$ and the site-resolved steady-state value of the $z$ spin using the parameters $\gamma_{1}=0, \gamma_{2}=0.024 \eta$ (red), $\gamma_{1}=0.12 \eta, \gamma_{2}=0$ (blue), and $\gamma_{1}=0.12 \eta, \gamma_{2}=0.024 \eta$ (purple). Here the steadystate $z$ component of spin on each site is computed by averaging its expectation value over the time-window $2200 \eta^{-1}<t<$ $2400 \eta^{-1}$. The first parameter set results in $\Gamma=0.6 \eta, \tau=0.02 \eta, \Gamma \tau=0.01$, and $2 \Gamma^{2} \tau=0.015 \eta$, whereas the last parameter set results in $\Gamma=1.2 \eta, \tau=0.02 \eta^{-1}, \Gamma \tau=0.026$, and $2 \Gamma^{2} \tau=0.063 \eta$ (indicating that the universal Lindblad equation accurately describes the system's dynamics). For the last parameter set, we compute steady-state magnetization and energy currents $\bar{I}_{M}$ and $\bar{I}_{E}$ by averaging their expectation values over the time-window $2200 \eta^{-1}<t<2400 \eta^{-1}$. The resulting values are given by $\bar{I}_{E}=0.63 \eta^{2} \pm 0.07 \eta^{2}$ and $\bar{I}_{M}=2.2 \times 10^{-3} \eta \pm 2.4 \times 10^{-5} \eta$.

(a)

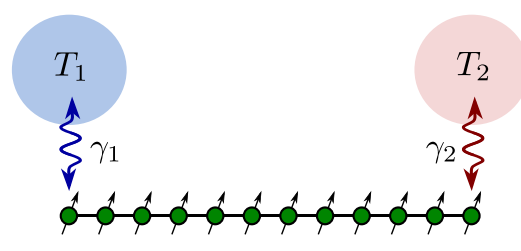

(b)

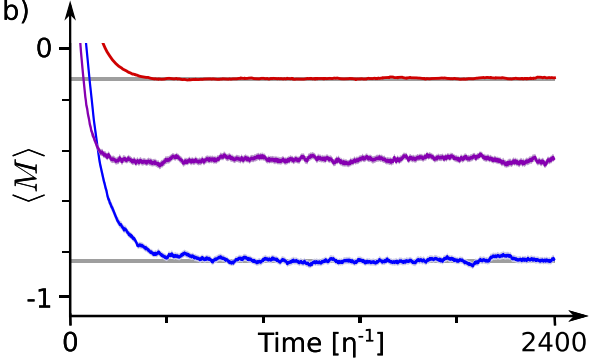

(c)

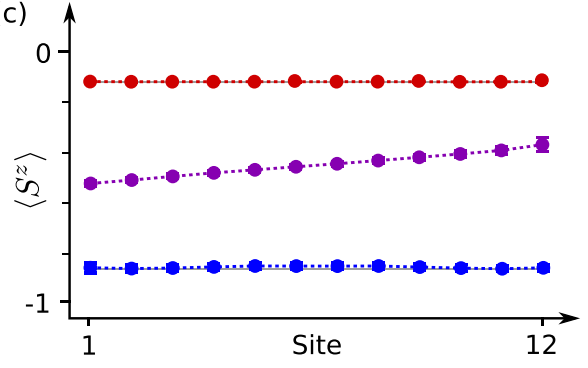

FIG. E2. Simulations of the open Heisenberg spin-chain model with parameter sets where the error bound computed with the corrected values of $\Gamma$ and $\tau$ guarantee the error of ULE to be small; this figure is complementary to Fig. 2 in the original paper. See the text for details and the original paper for further explanation of the figure. 